\title{
MAPAS, LENGUAJE Y Conceptos: Hacia unA TEORÍA PluRAlista DEL Formato DE LOS CONCEPTOS
}

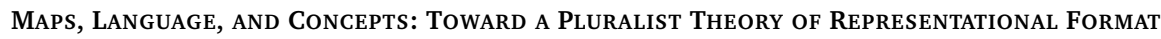

\author{
MARIELA AgUiLERA \\ CONICET/UNC, ARGENTINA \\ maguilera@ffyh.unc.edu.ar
}

\begin{abstract}
A great number of investigations suggest that cognition involves both linguistic and cartographic representations. These researches have motivated a pluralist conception of cognition; also, they have been used to clarify how maps differ from linguistic representations. However, the computational processes underlying the interphase between both kinds of representations deserve further attention. In this paper, I argue that, despite their differences, cartographic representations coexist and interact with linguistic representations in interesting ways.
\end{abstract}

Keywords: mental representations $\bullet$ predicative structure $\bullet$ mental vehicles $\bullet$ analogue $\bullet$ digital

\section{Introducción}

Comúnmente usamos y construimos distintos tipos de mapas. Éstos forman parte de nuestra vida cotidiana y, junto a las listas de supermercados, las cuentas, los organigramas y los recordatorios en nuestros teléfonos celulares, participan en el desempeño de distintas tareas cognitivas tales como la planificación de nuestro recorrido en auto o transporte público y la organización de nuestra jornada, por mencionar sólo algunas de ellas. Por otra parte, filósofos, psicólogos y neuro-científicos han sugerido que nuestra mente/cerebro almacena buena parte de la información en mapas cognitivos (Bermúdez 1998; Epstein, Patai, Julian \& Spiers 2017; Heck 2007; Hills, Jones, \& Todd 2012; O’Keefe \& Nadel 1978; Peacocke 1992b; Tolman 1948, entre otros). ${ }^{1}$ En particular, las investigaciones señalan que las habilidades para la navegación espacial observadas en las ratas, las abejas y mamíferos, entre ellos, los humanos, involucran algún tipo de sistema cartográfico (Boesch y Boesch 1984; Gould 1986; Gallistel 1998; Kamil \& Jones 1997, entre otros).

Sin embargo, frente al lenguaje, el papel en nuestra vida cognitiva de los demás tipos de representaciones es, con frecuencia, minimizado. En consonancia con 
una visión paradigmáticamente lingüística de nuestra cognición, una intuición filosófica predominante en la filosofía analítica y algunas de sus variantes cognitivas contemporáneas, ha sido que los conceptos tienen o presuponen algún tipo de medio lingüístico. Partiendo de dicha intuición, de la mano de las ciencias cognitivas, Fodor ha defendido que los conceptos son representaciones internas, es decir, particulares mentales, dotados de propiedades semánticas y sintácticas, sobre las cuales operan procesos computacionales. Dicho de otro modo, los conceptos son símbolos de un lenguaje del pensamiento.

Ahora bien, recientemente, algunos autores han sostenido que hay representaciones no lingüísticas que pueden jugar el rol de los conceptos (Camp 2007; Rescorla 2009b). Estos últimos trabajos señalan que muchos de los argumentos a favor del lenguaje del pensamiento presuponen condiciones que pueden ser satisfechas tanto por sistemas representacionales lingüísticos como cartográficos. Específicamente, se ha argumentado que los mapas cuentan con una estructura constitutiva adecuada para ciertos procesos inferenciales (Aguilera 2016, Camp 2007; Rescorla 2009b, 2009c).

Los abundantes trabajos y discusiones sobre mapas que tuvieron lugar en las últimas décadas han permitido clarificar cómo éstos se diferencian de otros tipos de representaciones, como las representaciones lingüísticas y las pictóricas (BraddonMitchell \& Jackson 1996; Camp, 2018; Heck 2007; Kulvicki 2015; Rescorla 2009a). ${ }^{2}$ Por otro lado, estos trabajos han contribuido al desarrollo de una concepción pluralista de la cognición, en tanto han mostrado que la cognición puede operar tanto sobre representaciones de tipo lingüístico como cartográfico. Sin embargo, en este marco pluralista, aun no se ha explorado cuáles son los procesos computacionales que ponen en relación el contenido de ambos tipos de representaciones. En ese sentido, el propósito de este trabajo es dar un paso en esa dirección y sostener que, pese a sus diferencias con las representaciones lingüísticas, las representaciones cartográficas pueden servir como vehículos de los conceptos e interactuar con las representaciones lingüísticas de modos muy interesantes. ${ }^{3}$

A diferencia de lo que plantean otras variantes de pluralismo (Haimovici 2018; McCaffrey 2015; Machery 2009; Weiskopf 2009a), la tesis defendida aquí trata sobre el formato de las representaciones o vehículos de los conceptos, antes que la estructura semántica o modalidad de la información representada por los conceptos. Como punto de partida, tomaré la tesis empírica de que nuestra cognición opera con representaciones mentales que tienen distintos tipos de formatos, particularmente, cartográficos y lingüísticos. Luego, sugeriré que algunas de las propiedades centrales del pensamiento conceptual pueden ser explicadas presuponiendo vehículos con un formato cartográfico. Para ello, tomaré como caso un mapa de subte, que instancia un tipo de sistema cartográfico altamente formal, que combina elementos simbólicos e icónicos. A partir de analizar ese caso, pretendo señalar algunas de las propieda- 
des formales de los mapas en general. En este sentido, mi estrategia metodológica es similar a la fodoriana, la cual se basa en el estudio de los rasgos formales de los sistemas lingüísticos para explicar posteriormente algunas propiedades de las representaciones mentales.

Ahora bien, la tesis de que los conceptos pueden ser vehiculizados por distintos tipos de formatos representacionales puede ser entendida de diferentes maneras. Por un lado, este tipo de pluralismo puede ser entendido como una propuesta sobre el formato de las representaciones de la mente animal, que permita explicar las similitudes y las diferencias entre el pensamiento animal y humano (Aguilera 2016, Camp, 2007; 2009; Rescorla 2009a; 2009b). Por otro lado, este tipo de pluralismo puede ser entendido como la tesis de que en nuestra cognición coexisten representaciones con distintos tipos de formatos. Entendido así, es preciso explicar cómo interactúan las representaciones que tienen formatos diferentes. Precisamente, en este trabajo, me centraré en esta variante de pluralismo según la cual en nuestra cognición coexisten conceptos vehiculizados por representaciones mentales con distintos formatos que, en virtud de sus propiedades formales, pueden interactuar entre sí de maneras muy interesantes.

A continuación, en la segunda sección, reviso algunos de los argumentos de Fodor a favor de la hipótesis del lenguaje del pensamiento. En la tercera sección, presento algunas razones para sostener que esos argumentos presuponen condiciones para las representaciones conceptuales que pueden ser satisfechas por sistemas representacionales tanto lingüísticos como cartográficos. En la cuarta sección, defenderé una concepción pluralista de los conceptos que, a diferencia de sus predecesoras, lejos de entender las representaciones cartográficas como aisladas de otros tipos de representaciones, se centra en el modo en que interactúan con representaciones lingüísticas, mediante transformaciones semánticas.

\section{La hipótesis del lenguaje del pensamiento}

De acuerdo con una visión filosófica estándar, los conceptos son los elementos constitutivos del pensamiento (Prinz 2002; Laurence \& Margolis 1999). Desde la perspectiva de la teoría representacional de la mente, esto significa que el pensamiento tiene una estructura interna combinatoria, en la que los conceptos funcionan como los componentes más básicos. Ello permite explicar rasgos centrales del pensamiento, tales como la composicionalidad, la aspectualidad y razonamiento.

Desde esta perspectiva, Fodor ha defendido la hipótesis del lenguaje del pensamiento; esto es, la idea de que los conceptos son representaciones mentales internas con un formato lingüístico (Fodor 1995; 2007; 2008, Fodor \& Pylyshyn 1988). Esta tesis descansa en una serie de argumentos y suposiciones. Primero, que el pensamien- 
to conceptual es composicional en tanto su contenido y su estructura sintáctica están determinados por el contenido y la estructura sintáctica de sus partes constitutivas. Esto implica que los conceptos que constituyen un pensamiento sean independientes del contexto representacional del que forman parte; pues un concepto conserva su aporte semántico y sus propiedades sintácticas en todos los pensamientos en los que ocurre sin verse afectado por la estructura representacional en la que se aloja (Fodor 1995, p. 16). Así pues, la composicionalidad se requiere para explicar la sistematicidad del pensamiento, esto es, el hecho de que "la habilidad para concebir un pensamiento implica la habilidad para concebir otros pensamientos con contenidos semánticos relacionados" (Fodor y Pylyshyn 1988, pág. 3) ${ }^{4}$. Esto implica que si una criatura es capaz de pensar que Juan ama a la chica, tiene los recursos conceptuales para pensar que la chica ama a Juan. La tesis de la sistematicidad suele sostenerse junto con la tesis de la productividad, según la cual los componentes del pensamiento han de poder ser recombinados para formar otros nuevos pensamientos. Conjuntamente, la sistematicidad y la productividad implican que los conceptos se pueden separar de los pensamientos de los que forman parte para ser recombinados y formar parte de otros pensamientos.

Segundo, los conceptos son aspectuales; representan las cosas de tal y cual manera, por ejemplo, a Fonta como un perro, a un objeto como una mesa (Fodor 2007), etc. Así pues, la aspectualidad puede entenderse en términos de la aplicación de predicados. Fodor (2007) y otros (Machery 2009) entienden el fenómeno de la aspectualidad en términos del contenido intencional o referencial: una representación es aspectual en tanto permite representar un ítem como perteneciendo a una clase determinada. ${ }^{5}$ Tercero, los conceptos juegan un rol inferencial; esto es, deben poder ser iterados a los fines de ser parte de cadenas de razonamiento. Así, por ejemplo, en el razonamiento Todos los hombres son mortales, Sócrates es un hombre, entonces Sócrates es mortal, la iteración del predicado HOMBRE permite concluir que Sócrates es mortal, encadenando los tres pensamientos en términos de premisas y conclusión. A partir de estas propiedades del pensamiento, Fodor ha argumentado que sólo representaciones con un formato lingüístico pueden jugar el rol de los conceptos, pues supone que la sistematicidad es exclusiva de este tipo de sistemas y sólo las oraciones tienen predicados, los cuales son requeridos para dar cuenta tanto de la aspectualidad y del rol inferencial de los conceptos.

Ahora bien, como adelanté arriba, distintos tipos de sistemas representacionales pueden dar lugar a las propiedades que Fodor atribuye a los conceptos. En particular, argumentaré que ciertos tipos de sistemas cartográficos son composicionales. La composicionalidad de los mapas ha sido defendida previamente por Camp (2007) y Rescorla (2009b). A diferencia de ellos diré que además tienen una estructura predicativa. Al tener una estructura predicativa, estos sistemas son aspectuales y pueden funcionar como vehículos adecuados para las transiciones inferenciales. Si esto es 
así, la hipótesis del lenguaje del pensamiento, aun cuando permita explicar distintos fenómenos del pensamiento conceptual, no constituye una caracterización excluyente de los conceptos. Pues, los argumentos a favor de esta hipótesis son compatibles con la posibilidad de que otros sistemas representacionales no lingüísticos sean conceptuales.

\section{La hipótesis de los mapas mentales}

Uno de los supuestos sobre los que descansa la hipótesis del lenguaje del pensamiento es la tesis de que los estados mentales involucran representaciones mentales con una estructura interna combinatoria. Esto equivale a decir que los estados mentales son el producto de la realización de operaciones computacionales a partir de un conjunto limitado de elementos básicos, con propiedades sintácticas y semánticas, los cuales se pueden recombinar para dar lugar a otros estados mentales, con distintos contenidos. A partir de esta tesis, Fodor concluye que las representaciones que juegan el rol de conceptos es lingüístico (Fodor 1975, 2007, 2008; Fodor \& Pylyshyn 1988).

Aun cuando aceptemos esta imagen de la cognición, es cuestionable que ese tipo de estructura sea privativa de las representaciones con formato lingüístico. Así, por ejemplo, Camp (2007) ha dado razones contundentes para negar la tesis de que todo sistema que cuente con una estructura interna combinatoria sea un lenguaje. A partir de los mapas de ruta, ella muestra que los sistemas cartográficos tienen una estructura interna combinatoria y que se diferencian de los sistemas lingüísticos debido a explotar un isomorfismo espacial. Por otro lado, Camp (2007) sostiene que los mapas y los diagramas, al igual que el lenguaje, están formados por elementos formales que se combinan de acuerdo con reglas sistemáticas. Por esa razón, sostiene que podrían funcionar como los vehículos de inferencias formales (pág. 154). Similarmente, Rescorla (2009b) sostiene que hay procesos racionales que operan en sistemas cartográficos. Para Rescorla, estos sistemas son sistemáticos y productivos, al igual que los lenguajes. Sin embargo, niega que tengan una estructura predicativa $\mathrm{y}$, por tanto, una forma lógica. Aduce que los mapas se caracterizan por su forma geométrica, por lo cual - continúa - hay procesos racionales que no requieren una forma lógica ni vehículos lingüísticos.

Se podría decir que en tanto los mapas tienen una estructura combinatoria tienen, después de todo, un formato lingüístico (ver Blumson 2012). Para responder esta objeción, Camp (2007, p.152) diferencia la hipótesis débil del lenguaje del pensamiento, de la hipótesis fuerte. De acuerdo con la formulación débil, la HLP implica que el pensamiento requiere un sistema de vehículos representacionales con algunos elementos recurrentes que puedan ser recombinados de acuerdo con algunas reglas para producir otros contenidos. La formulación fuerte, en cambio, implica que el pen- 
samiento requiere una estructura sintáctica y semántica específicamente lingüísticas. En las discusiones actuales, esto suele implicar que el lenguaje del pensamiento es analizable en términos del cálculo de predicados y se compone, por tanto, de conectivas lógicas y cuantificadores (Fodor 2008, 2007; Rescorla, 2009b). La hipótesis de que el pensamiento puede tener un formato cartográfico se opone a la formulación fuerte - que es la que parece estar defendiendo el mismo Fodor $(2007,2008)-y$ explota la idea de que hay diferencias explicativas significativas entre distintos formatos representacionales.

Al igual que los lenguajes, los mapas están constituidos por distintos tipos de elementos que representan con un alto grado de arbitrariedad distintos tipos de entidades. Sin embargo, a diferencia de lo que sucede en los lenguajes, los sistemas cartográficos se caracterizan por explotar algún tipo de isomorfismo espacial: ${ }^{6}$ en los mapas, la disposición espacial de sus elementos es isomorfa a la disposición espacial de las entidades representadas. En este sentido, los mapas son sistemas representacionales híbridos, pues emplean elementos simbólicos, semánticamente arbitrarios, como las palabras, y elementos icónicos, como las líneas, que descansan en algún tipo de isomorfismo. Cabe agregar que distintos tipos de mapas explotan distintos tipos de isomorfismos. Así, por ejemplo, los mapas de ruta explotan relaciones métricas para representar a escala la distancia entre objetos en el espacio. Los mapas físicos, en cambio, explotan propiedades cromáticas para representar el relieve de una superficie, a una escala definida. Siguiendo a Camp y Rescorla, diré que el formato de las representaciones cartográficas es adecuado para formar parte de los procesos computacionales característicos de los conceptos. Sin embargo, a diferencia de ellos, diré que los mapas tienen una estructura predicativa. Para ello, adoptaré una noción de predicado más básica que la provista por la lógica de primer orden. Ello permitirá caracterizar, en la sección 4, la naturaleza de los vínculos semánticos entre los mapas y las oraciones. En lo que sigue, me centraré en una variedad de mapas que goza de un gran nivel de abstracción e idealización: los mapas de subte. A partir del análisis de sus propiedades formales, argumentaré que los mapas tienen una estructura predicativa primitiva fuertemente composicional. Esto permite sugerir que representaciones mentales con este tipo de formato podrían jugar el rol de conceptos.

Los mapas de subte pertenecen a los sistemas topológicos, que se encuentran a mitad de camino entre los sistemas métricos, como los mapas de ruta, y los diagramáticos, como los diagramas de Venn. Al igual que los mapas de ruta, los mapas de subte explotan un isomorfismo espacial y, al igual los diagramas, explotan relaciones altamente idealizadas y abstractas. Más específicamente, los sistemas topológicos explotan relaciones de convergencia, conectividad y continuidad. Esto quiere decir que la disposición espacial de cada uno de los elementos del mapa representa relaciones topológicas entre las entidades representadas. En este sentido, una vez que un elemento es introducido en este tipo de mapas, formará parte de una red de relaciones 
topológicas. Por otro lado, estos mapas se componen de elementos recurrentes que se pueden recombinar conforme ciertas reglas de combinación, dando lugar a distintas estructuras representacionales y preservando sus propiedades semánticas en las diferentes estructuras representacionales en las que aparecen (Camp 2007). De esta manera, estos mapas pueden expresar diferentes contenidos por medio de distintas combinaciones de ejemplares de los mismos tipos de elementos. En este sentido, se trata de representaciones no lingüísticas que, al igual que las oraciones, tienen una estructura interna combinatoria y constituyen, junto a los mapas de ruta, un contraejemplo a la tesis de Fodor, pues cumplen con las condiciones mínimas para jugar el rol de los conceptos. Este tipo de estructura es, además, composicional, pues el significado del mapa - esto es, de la representación tomada como un todo - depende del contenido o significado de los elementos que lo constituyen.

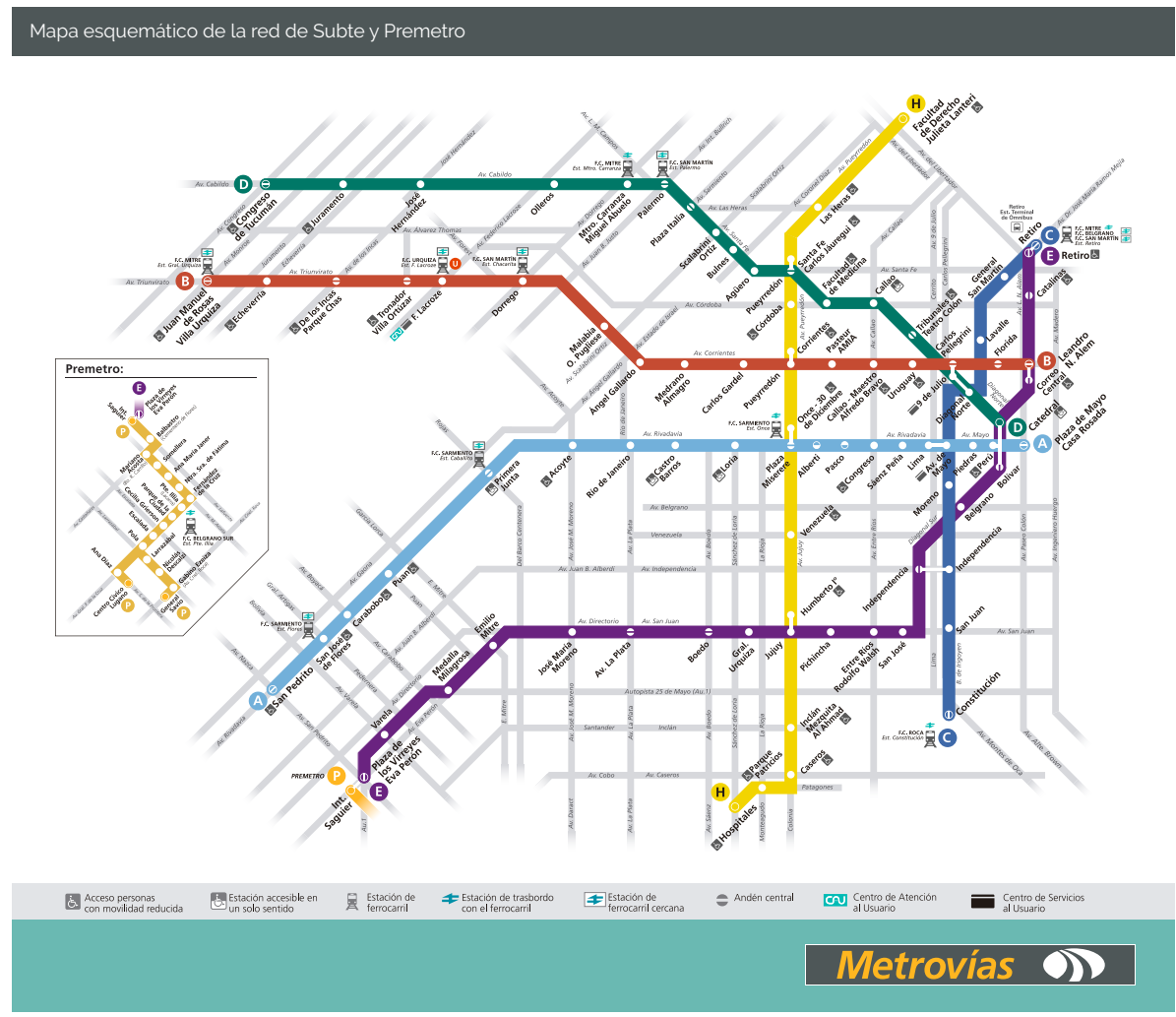

Figura 1: Mapa del metro de CABA. Fuente: http://www.buenosaires.gob.ar.

Finalmente, entre los elementos de los mapas de subte, se pueden distinguir elementos generales y elementos singulares. Así, por ejemplo, en la figura 1, los círculos representan la propiedad de ser una estación de conexión y las líneas representan la 
propiedad de ser una línea de subte, entre otros. Se trata, pues, de elementos que pueden utilizarse sistemáticamente para atribuir propiedades generales a distintas entidades. Los nombres, en cambio, designan estaciones particulares y los colores de las líneas y las letras, recorridos de subte particulares, de modo que tienen una función singularizadora. En este sentido, mi tesis es que estos mapas tienen una estructura predicativa, pues tienen los elementos necesarios para atribuir propiedades generales a entidades particulares. Por esta razón, los mapas son representaciones aspectuales y tienen una estructura adecuada para las transiciones inferenciales.

\section{Mapas, predicados y composicionalidad}

Para determinar si las representaciones cartográficas pueden desempeñar el rol de los conceptos, es central analizar si tienen una estructura predicativa, pues ello garantizaría que puedan dar cuenta de la aspectualidad y de la racionalidad, centrales al rol de los conceptos. La aspectualidad es una característica de los conceptos, y alude al hecho de que cuando pensamos en algo, lo hacemos pensándolo como perteneciendo a una clase determinada, en virtud de presentar ciertos aspectos. Esto es, en el pensamiento las entidades son representadas bajo ciertos conceptos. De acuerdo con Fodor, para dar cuenta de la aspectualidad todo lo que se requiere es una estructura predicativa, que permita que una entidad sea representada de tal y cual manera. De esta forma, representar una entidad, a, de cierta manera, como $\mathrm{x}$, equivale a representar esa entidad, a, como cayendo bajo el concepto X.

Por lo que representar (por ejemplo, mentalmente) a Mr James como un gato es representarlo como cayendo bajo el concepto GATO; y representar a Mr James como cayendo bajo el concepto GATO es representarlo como un gato; y pensar en Mr James como un gato requiere aplicar el concepto GATO a Mr James. (Fodor 2007, p.105. La traducción es mía.)

De modo que evaluar si los sistemas cartográficos son aspectuales equivale a preguntar si tienen una estructura predicativa. Esto implica que algunos elementos han de operar como predicados mientras que otros han de operar como nombres, esto es, términos o íconos singulares.

Casati y Varzi (1999) han defendido que los mapas tienen una estructura predicativa. Esta propuesto ha recibido numerosas críticas. Precisamente, para negar que los mapas tengan predicados, Rescorla analiza la propuesta de Casati y Varzi y construye un argumento basado en la intuición de la ausencia. Según esta intuición, la ausencia de un elemento en un mapa representa la ausencia del objeto representado por ese elemento (Casati \& Varzi 1999). De acuerdo con Rescorla, esta intuición es incompatible con la noción clásica de predicado, tal como es entendida a partir de los desarrollos de Frege y Tarski. Broner (2015) y Camp (2018), sostienen, sin embargo, 
que esta intuición no se sostiene para muchos mapas. No obstante, Camp (2018) da otras razones para negar que los mapas tengan predicados. Por su parte, tratando de responder al argumento de Rescorla, Kulvicki (2015) defiende una manera de adaptar la noción clásica de predicado, para los mapas de modo tal que se pueda dar cuenta de la intuición de la ausencia. ${ }^{7}$

Es de notar que pese a sostener en algunos casos posiciones contrapuestas, en su mayoría, los participantes del debate adhieren a una concepción de los predicados basada en el lenguaje de primer orden (o cálculo de predicados) y los desarrollos de Frege y Tarski. Esta concepción exige, entre otras cosas, la presencia de conectivas lógicas y cuantificadores. Sin embargo, creo que tomar la noción de predicados provista por la lógica de primer orden presupone la cuestión a favor de una concepción lingüística de los conceptos. En este sentido, coincido con Fodor, Heck, Rescorla, etc., en que los mapas no pueden alojar cuantificadores, centrales al cálculo de predicados.

En lugar de ofrecer una respuesta a Camp (2018) y Rescorla (2009c), en este trabajo, propongo usar una noción de predicados más básica que la derivada del cálculo de predicados. Como veremos más adelante, esta estrategia permitirá explicar el modo en que interactúan semánticamente representaciones cartográficas y lingüísticas. Para establecer si un sistema representacional tiene una estructura predicativa, adoptaré una concepción primitiva de los predicados y expresiones singulares. De acuerdo con esta caracterización, un elemento es singular si tiene una pretensión referencial singularizadora. Por su parte, un elemento es un predicado si tiene una función general atributiva; esto es, si atribuye sistemáticamente la misma propiedad a entidades particulares. De esta manera, tomando la caracterización de Taylor (2010, p.82), si dos ejemplares del mismo tipo de símbolo refieren explícitamente a un mismo objeto y son referencialmente independientes de los ejemplares de cualquier otro tipo, entonces, se trata de un nombre propio. Caso contrario, si los distintos ejemplares de un mismo tipo de símbolo no son co-referenciales entre sí, el símbolo funciona como predicado.

La idea es que una representación tiene una estructura predicativa si posee los elementos necesarios para atribuir de manera sistemática propiedades generales a entidades particulares. Es fácil ver como los mapas tienen este tipo de componentes. Veamos. Por ejemplo, PALERMO, en la figura 1, funciona como una expresión singular, pues tiene una función singularizadora, de designar una entidad particular. Si nos encontráramos además con la siguiente aclaración al margen del mapa: Palermo permanecerá cerrada los días domingos y feriados, habremos de suponer que "Palermo" refiere a la misma estación que el ejemplar utilizado en el mapa, de modo que la información pueda ser explotada eficazmente. Los distintos ejemplares (tokens) del círculo blanco (type), en cambio, funcionan como elementos predicativos, pues representan una propiedad general que puede ser atribuida a distintas 
entidades particulares. En otras palabras, los distintos ejemplares del símbolo que denota estaciones, $\bigcirc$, son referencialmente independientes entre sí. La combinación de estos elementos, por su parte, le atribuye a Palermo la propiedad de ser una estación, subsumiendo a PALERMO bajo ESTACIÓN. En este sentido, al permitir combinar representaciones de propiedades con representaciones de particulares, los mapas cuentan con los recursos mínimos para la aspectualidad.

Por otra parte, al explotar un isomorfismo espacial, los mapas representan relaciones espaciales por medio de la disposición espacial de sus elementos constitutivos. Esto quiere decir que cada vez que un elemento constitutivo es introducido en un mapa, éste es inmediatamente incorporado a una red de relaciones espaciales junto con otros elementos del mapa. Así, por ejemplo, en tanto forma parte de una estructura topológica, el elemento singular CONSTITUCIÓN forma parte de una red de relaciones espaciales, en tanto Constitución es representada como conectada con San Juan, Independencia y demás. Lo mismo ocurre con los elementos generales. Así, por ejemplo, al ser insertados en un mapa, distintos ejemplares del símbolo círculo, que representan la propiedad de ser una estación, también ocuparán una posición en la red topológica. De esta manera, los elementos constitutivos del mapa funcionarían como los argumentos de relaciones espaciales como conectividad, convergencia y contigüidad (Aguilera \& Castellano, manuscrito). En otras palabras, los mapas de subte, como una variedad de sistema cartográfico, explotan tanto predicados de aridad 1 como de aridad 2.

Veamos ahora si los mapas son adecuados para cumplimentar un rol inferencial, característico de los conceptos. Si nos remitimos a la tradición filosófica, veremos que se ha adoptado, por lo general, una concepción lingüística de la inferencia. Como ejemplo, veamos la siguiente cita:

Nosotros entendemos las inferencias en términos formales - en términos de reglas que operan sobre representaciones en virtud de su estructura. Pero no tenemos una teoría de transiciones inferenciales entre pensamientos que no tengan vehículos lingüísticos (Bermúdez 2003, p.111. La traducción es mía).

Sin embargo, un mayor acercamiento a sistemas alternativos, como el caso de los mapas y de los diagramas, rápidamente pone en la mira esta concepción. En esta dirección, Camp (2007) sostiene que, con ciertos agregados, los mapas de ruta sirven como sistemas dinámicos para el razonamiento deductivo. Rescorla (2009a), en cambio, sostiene que los mapas poseen una estructura sintáctica apropiada para procesos de inferencias inductivas. Dado que adhiere a una concepción de los predicados basado en el lenguaje de primer orden, Rescorla niega que los mapas tengan predicados y que puedan participar en inferencias deductivas.

Por cuestiones de espacio, no me detendré en este punto aquí. Diversos trabajos han mostrado que los mapas tienen los recursos para hacer inferencias (Agui- 
lera 2016, Camp 2007, Rescorla 2009a). En particular, los mapas se componen de elementos con funciones semánticas y sintácticas heterogéneas, que contribuyen a determinar su valor semántico. Además, dado que - como he argumentado — los mapas tienen una estructura predicativa, cuentan con los elementos requeridos para participar en algunas transiciones inferenciales formales. Consecuentemente, el formato de los mapas es adecuado para dar cuenta de la racionalidad, característica del pensamiento conceptual. Así, por ejemplo, la iteración de círculos blancos permite concluir que Palermo y Bulnes están conectados. A partir de la combinación de distintos ejemplares del círculo blanco y líneas, se puede inferir que, si hay una conexión entre las líneas verde y roja, y entre la roja y la celeste, entonces las líneas verde y celeste están conectadas, etc. (ver figura 2).
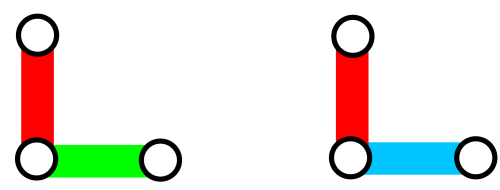

Figura 2: Fragmentos de un mapa de subte inspirado en el mapa de subte porteño. Ver figura 1.

Podría objetarse que estos ejemplos no involucran transiciones inferenciales, puesto que la información está explícitamente representada en el mapa. Pero entonces, por las mismas razones, también deberíamos negar que " $P x \wedge Q x)$ entonces $Q x$ " constituye una inferencia. De modo que la objeción no se sigue. Por otro lado, como veremos más adelante, el hecho de que se puedan distinguir el rol sintáctico y el aporte semántico de los elementos de un mapa permite explicar la existencia de transiciones semánticas entre los mapas y otros tipos de representaciones. Esto posibilita que los mapas sean combinados con representaciones con otros formatos, incrementando considerablemente su potencial inferencial (Aguilera \& Castellano, manuscrito).

\subsection{Mapas, composicionalidad y flexibilidad}

¿Son composicionales los mapas? Algunas veces, el carácter composicional de las representaciones cartográficas ha sido puesto en duda, argumentando que, al igual que las representaciones pictóricas, los mapas tienen lo que se denomina flexibilidad semántica (Heck 2007). Una representación tiene flexibilidad semántica cuando el aporte semántico de las partes que la componen cambia a partir de su relación con las otras partes de la representación (Braddon-Mitchell \& Jackson 1996; Recabarren, manuscrito). Así pues, si una representación exhibe esta propiedad, entonces no es 
composicional y, por tanto, tampoco puede funcionar como vehículo de los conceptos. Por lo general se acepta que las representaciones pictóricas y la percepción, al menos cuando es explicada bajo un modelo pictórico, exhiben flexibilidad semántica de un modo paradigmático (Heck 2007). Así, por ejemplo, un mismo vestido a rayas puede verse como negro y azul o blanco y dorado, dependiendo de la iluminación y los colores del contexto. Similarmente, en la figura 3, la misma figura negra es interpretada como elíptica y como circular, debido a las diferencias entre los elementos del contexto, en A y B (Recabarren, manuscrito).

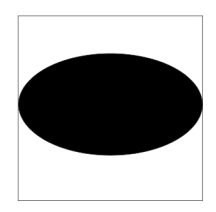

A

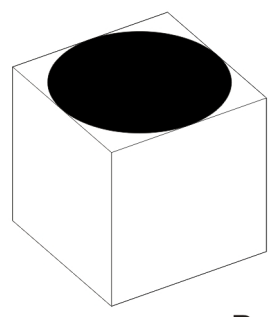

B

Figura 3: Elipsis vs. Círculo.

Sin embargo, la flexibilidad semántica no sólo es una propiedad de las representaciones pictóricas, sino que se extiende también al lenguaje natural (Travis 2008; Camp 2015). Veamos un conocido ejemplo de Searle (1992):

- Juan corta el pasto

- María corta la torta

- María se corta el pelo

Aquí, el significado de "cortar" varía en virtud de los contextos oracionales en los que aparece. Por lo general, este tipo de variaciones es explicado en algunos casos como producto de la interacción de las palabras con las que una expresión aparece - el contexto intra-lingüístico - y en otros, en términos del contexto externo en el que la oración es proferida (Recanati 2012; Travis 2008; Clapp 2012). De modo tal que la flexibilidad semántica no sólo atenta contra la sistematicidad de las representaciones pictóricas, sino también contra la sistematicidad del lenguaje natural (Johnson 2004). A partir de este tipo de consideraciones, Fodor (2001) ha defendido una concepción mixta de la sistematicidad, pues sostiene que mientras los lenguajes naturales no son sistemáticos ni composicionales, el lenguaje del pensamiento sí lo es. Por esa razón, Camp (2015) sostiene que la hipótesis del lenguaje del pensamiento ha de apoyarse en lenguajes formales, como el cálculo de predicados, antes que en lenguajes naturales. ${ }^{8}$ Habría que ver, no obstante, si la flexibilidad semántica constituye un 
defecto o una virtud de los lenguajes naturales, y si querer excluirla de nuestra caracterización del pensamiento no constituye una visión demasiado restrictiva de la composicionalidad (Aguilera 2016). Pero por cuestiones de espacio no me ocuparé de esto aquí. Por mor del argumento, aceptaré la tesis de la composicionalidad tal como Fodor la entiende.

Algunos autores han argumentado que los mapas tienen flexibilidad semántica, pues consideran que el aporte semántico de las partes de un mapa cambia en relación con las otras partes que lo componen (Braddon-Mitchell \& Jackson 1996; Heck 2007). Si los mapas tuvieran esta propiedad, no serían composicionales en el sentido que nos interesa aquí y, por tanto, no podrían ser conceptuales. Analicemos el mapa de subte porteño (ver figura 1). En este mapa, por ejemplo, distintos ejemplares (tokens) de círculo $\bigcirc$ representan la propiedad de ser una estación. Al combinarse con CONSTITUCIÓN, Constitución es representada como una estación. Pero el mapa no sólo representa eso, sino que también representa a Constitución como la última estación - o estación terminal final — de la línea $C$ en virtud de la posición que ocupa el ejemplar de círculo utilizado para representar Constitución. Sin embargo, al ocupar otra posición, otros ejemplares del mismo tipo de símbolo ya no representan la ÚLTIMA ESTACIÓN, sino sólo una ESTACIÓN-CONECTADA a otras estaciones. Así, por ejemplo, Independencia es representada como una estación conectada a Moreno y San Juan por la línea celeste.

Ahora bien, lo que quiero defender aquí es que los aparentes cambios semánticos sufridos por los sistemas cartográficos no son de la misma naturaleza que la flexibilidad semántica exhibida por las imágenes, el lenguaje natural y la percepción. Por el contrario, al menos en ciertos casos, estos cambios pueden explicarse apelando a la carga semántica de las reglas de combinación del propio sistema. Volviendo al ejemplo anterior, el mapa de subte constituye una variedad de sistema topológico. Este tipo de sistema explota un isomorfismo espacial formal y abstracto, específicamente, explota relaciones de conectividad. Como tal, cada uno de los elementos que forma parte del mapa, también forma parte de una red de relaciones topológicas, representadas de manera sistemática mediante la combinación de elementos constitutivos del mapa. Ahora bien, el tipo de relaciones espaciales representadas por un mapa dependerá de las reglas combinatorias del sistema al que pertenece el mapa. En otras palabras, en nuestro ejemplo, las relaciones topológicas entre los elementos del mapa espejan relaciones topológicas entre las entidades representadas por dichos elementos. De esta manera, cuando una estación es representada en el mapa, aparece conectada a otras estaciones y líneas representadas. Cuando sólo se encuentra conectada unidireccionalmente a otras estaciones, será representada como estación terminal final. Dicho de otra forma, propiedades tales como estación terminal final, estar conectada, etc. son atribuidas sistemáticamente a las entidades representadas por el mapa en virtud de sus reglas de combinación (Aguilera \& Castellano, manuscrito). 
Podría argumentarse que esto mismo sucede en el caso de las representaciones lingüísticas, u oraciones, cuyo contenido es determinado por el contenido de los elementos que lo constituyen y las reglas de combinación del lenguaje al que pertenecen. Hay, sin embargo, una importante diferencia: en el caso de las oraciones, las reglas de combinación determinan el contenido oracional de una manera exclusivamente sintáctica. En cambio, en el caso de los mapas, las reglas combinatorias contribuyen al contenido representacional no solamente de una manera sintáctica, sino también explotando propiedades semánticas. Dado que la disposición espacial de los elementos constitutivos del mapa representa la disposición espacial de los objetos representados por tales elementos, la disposición espacial de los elementos del mapa opera como un predicado relacional o de aridad > 1: i.e. CONECTADO (ESTACIÓN INDEPENDENCIA, LÍNEA CELESTE, ESTACIÓN SAN JUAN (ver figura 4)).

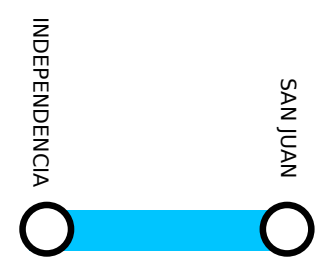

Figura 4: Fragmento de un mapa de subte inspirado en el mapa de subte porteño (figura 1).

Esto constituiría un importante rasgo de los mapas, pues debido a explotar un isomorfismo espacial, las reglas combinatorias de los mapas - a diferencia de las reglas combinatorias características de los lenguajes - poseen propiedades sintácticas y semánticas para determinar el contenido del mapa. En este sentido, mientras que el lenguaje natural exhibe flexibilidad semántica, en tanto el contenido de las palabras depende de los contextos tanto intra como extra-lingüísticos, los mapas serían fuertemente composicionales, en tanto el contenido de un mapa depende del aporte semántico de los elementos que lo componen y del aporte semántico y sintáctico de sus reglas de combinación.

Ahora bien, decir que los mapas tienen una estructura predicativa composicional no implica que no haya diferencias entre los sistemas lingüísticos y los cartográficos. Por empezar, como sostuve arriba, mientras que, desde la perspectiva de la TRM, las reglas de combinación de los sistemas lingüísticos determinan el contenido de las representaciones complejas, explotando exclusivamente propiedades sintácticas, los sistemas cartográficos se caracterizan por reglas combinatorias que explotan propiedades semánticas y sintácticas. Adicionalmente, al explotar un isomorfismo espacial entre la representación y lo representado, los sistemas cartográficos ven restringido el tipo de entidades y relaciones que pueden representar, así como sus posibilida- 
des de combinación. ${ }^{9}$ Por ejemplo, los mapas sólo pueden expresar pensamientos singulares. La razón es que, al representar una propiedad en un mapa, la misma se representa como instanciada por entidades particulares, pues la ubicación de un elemento en un mapa implica que la entidad representada por ese elemento ocupa una posición espacial determinada. En otras palabras, los predicados de los mapas siempre se aplican en contextos referenciales. Así, la instanciación del círculo, $\bigcirc$, en un mapa de subte no puede más que representar la propiedad ser una estación de subte instanciada por una estación de subte particular, por ejemplo, Independencia. Se podría objetar que un mapa puede representar todas las entidades relevantes de una cierta clase - por ejemplo, todas las estaciones de subte de una ciudad. Sin embargo, ello no implica que el mapa tenga los recursos para representar pensamientos generales sobre ellas - i.e. que todas las estaciones tienen la propiedad $\mathrm{X}$ o que existe una estación con la propiedad P. Para ello, se necesitan recursos lógicos como los cuantificadores, no disponibles en un mapa (Aguilera \& Castellano, manuscrito). Hay pensamientos generales que pueden estar en la base de nuestra interpretación de un mapa o bien pueden ser extraídos de él. Pero éstos deben ser expresados por otro tipo de representaciones; i.e. lingüísticas.

\section{Sistemas cognitivos plurales}

En la sección previa, argumenté que representaciones con formato cartográfico podrían satisfacer las condiciones mínimas requeridas para dar cuenta de la aspectualidad, la composicionalidad y la racionalidad. Por esa razón, este tipo de representaciones sería buen candidato para jugar el rol de los conceptos. Sin embargo, también vimos que los mapas tienen algunas restricciones expresivas y combinatorias. Precisamente, creo que la hipótesis de los mapas mentales podría dar cuenta de la cognición y del razonamiento animal, su alcance y limitaciones. Pero ésta no es la idea que me interesa explorar aquí. Mi propuesta, en cambio, es desplazar la atención hacia representaciones cartográficas que interactúan con otros tipos de representaciones, como sucedería en el caso de la cognición humana, en la que coexisten tanto mapas cognitivos como representaciones lingüísticas. Como veremos en esta sección, en virtud de su formato, los mapas pueden interactuar con representaciones lingüísticas de modos muy interesantes.

Los distintos avances teóricos sobre los mapas mencionados atrás diluyen la intuición extendida de que el pensamiento es esencialmente lingüístico. Esa intuición obtura una imagen de la cognición - impulsada por filósofos, psicólogos y neurocientíficos - que es tan plausible como luminosa. De acuerdo con esta imagen, nuestro pensamiento es representacionalmente rico en virtud de albergar vehículos representacionales con distintos formatos. No obstante, para delinear esta imagen de 
la cognición, es preciso explorar de qué manera se relaciona el contenido de las representaciones cartográficas con el de las representaciones lingüísticas. Puesto que, como es de esperar, estos distintos formatos representacionales no sólo coexisten, sino que además interactúan de modos muy interesantes. En lo que sigue, continuaré usando el mismo tipo de estrategia metodológica, analizando la relación entre mapas y oraciones concretas como una aproximación para pensar en los modos de interacción que podrían tener las representaciones mentales si tuvieran distintos tipos de formatos. Si bien se trata de una investigación puramente teórica y especulativa, constituye una herramienta útil para articular dentro de una teoría representacional de la mente, el racimo de teorías psicológicas, filosóficas y neuro-científicas que sostienen que nuestra cognición opera con representaciones cartográficas (Bermúdez 1998; Epstein, Patai, Julian, \& Spiers 2017, Heck 2007; Hills, Jones, \& Todd, 2012; O’Keefe \& Nadel 1978; Peacocke 1992b; Tolman 1948).

Si bien en los últimos años el estudio de los mapas y de las imágenes ha tenido un importante lugar en la agenda filosófica, la relación que habría entre este tipo de representaciones y las oraciones ha sido poco o nada explorada. Hay, no obstante, algunas excepciones. Por ejemplo, Beck (2013), en un intento por explicar por qué no podemos especificar el contenido del pensamiento animal, sostiene que el pensamiento de los animales tiene un formato analógico. Este tipo de representaciones se caracteriza por estar sujeto a la ley de Weber; por esa razón exhibe fallos en la sistematicidad: dado que las representaciones numéricas analógicas son isomorfas a sus referentes, se vuelven indiscriminables a medida en que su ratio se aproxima (pág. 539). Como la sistematicidad es un rasgo innegociable de los conceptos, Beck concluye que este tipo de representaciones no son conceptuales. Lo que quisiera destacar aquí es que, por medio de la hipótesis del formato de las representaciones, Beck pretende dar cuenta de la dificultad que tenemos para expresar lingüísticamente el contenido del pensamiento animal. Su hipótesis es que las representaciones con diferentes formatos no pueden ser traducidas (Beck 2013, p.526). De esta manera, plantea una suerte de escepticismo para especificar lingüísticamente el contenido de las representaciones analógicas, que no sólo están presente en la cognición animal, sino que además forman parte de nuestra cognición básica (Carey 2009). Heck (2007), en cambio, ha presentado una propuesta más optimista. Si bien considera que, al igual que las representaciones perceptivas, los mapas son no conceptuales, interactúan racionalmente con nuestras creencias, las cuales están vehiculizadas por representaciones con formato lingüístico. Sin embargo, poco nos dice acerca de cómo serían las relaciones lógicas entre representaciones cartográficas y lingüísticas.

En contraste con estas propuestas, mi hipótesis es que al tener una estructura predicativa composicional, representaciones con formato cartográfico podrían servir como vehículos de los conceptos. Esta hipótesis permite dar una aproximación de los vínculos semánticos entre las representaciones cartográficas y lingüísticas. En este 
sentido, las dificultades debidas a las diferencias de formato que pudieran surgir en la traducción de un sistema cartográfico a otro lingüístico no tendrían por qué llevarnos a consecuencias escépticas, como las que Beck señala. Este desideratum surge de la idea, presentada en las secciones previas, de que los conceptos son postulados, entre otras cosas, para explicar los procesos de razonamiento. Por esta razón, es importante comprender cuál es la naturaleza de los procesos computacionales que ponen en relación el contenido de ambos tipos de representaciones. En otras palabras, si hemos de aceptar que nuestra cognición opera con representaciones con distintos tipos de formatos, es una condición que tanto las representaciones cartográficas como las lingüísticas puedan interactuar en los procesos cognitivos dinámicos, propios de los conceptos. En esa dirección, en "Heterogeneous inference with maps" (Aguilera \& Castellano), sostuvimos que los mapas podían participar en inferencias heterogéneas, en las que se combinan representaciones lingüísticas y cartográficas. En este trabajo, me centraré en una cuestión más básica, relacionada con las transiciones entre los contenidos de los mapas y las oraciones.

Para avanzar en esta hipótesis, tomaré la distinción clásica propuesta por Dretske entre representaciones analógicas y representaciones digitales. ${ }^{10}$ Mi hipótesis es que los mapas poseen un adecuado equilibrio entre lo analógico y lo digital, el cual les permite mantener relaciones semánticas y lógicas con las oraciones, sin tener el mismo tipo de formato que ellas.

Diré que un signo (estructura, evento, estado) lleva información de que $s$ es $F$ en forma digital si y sólo sí no lleva información adicional sobre $s$, información que ya no esté contenida en $s$ siendo $F$ (Dretske 1981, p.26. La traducción es mía).

Si el signo lleva información adicional acerca de $s$, información que no esté contenida en $s$ siendo $F$, entonces diré que el signo lleva esta información en forma analógica. Cuando un signo lleva la información de que $s$ es $F$ en forma analógica, siempre lleva información sobre $s$ más específica y más determinada que el que sea $F$ (Dretske 1981, p.26. La traducción es mía).

Teniendo en cuenta esta distinción, diré que las oraciones son representaciones que exhiben un alto grado de digitalidad, pues pueden transmitir información de una manera considerablemente más precisa y arbitraria que los mapas: pueden atribuir la propiedad $\mathrm{P}$ a una entidad sin transmitir, con ello, otra información. Por ejemplo, en "Cruz es una chica", el predicado atribuye únicamente información acerca del género. ${ }^{11}$ Esto puede suceder, en parte, gracias a que las palabras representan información de una manera extremadamente selectiva; esto es, pueden representar con precisión una propiedad determinada, sin hacer referencia a otras propiedades que su referente pudiera tener. Por otro lado, las palabras no sólo son arbitrarias en 
tanto su aporte semántico es independiente de relaciones de semejanza con lo representado (i.e. el hecho de que una palabra escrita en una oración ocupe un lugar en el espacio -en tanto marca material - no implica que aquello a lo que refiere ocupe un lugar en el espacio). Son arbitrarias, además, en tanto el modo en que se combinan las representaciones lingüísticas no involucra la atribución de otras propiedades y relaciones, salvo información sumamente abstracta sobre su papel lógico y el estatus ontológico de las entidades a las que se refiere (relativa a si se trata de propiedades, relaciones o particulares) (Camp 2004; Wittgenstein 1921). En consecuencia, el espacio lógico del que forman parte permite combinar conceptos con un alto grado de libertad.

En contraste, en un mapa, la atribución de una propiedad, P, involucra la atribución sistemática de otras propiedades o relaciones, Q, R, S. En particular, involucra la atribución de relaciones espaciales, constitutivas del sistema. En el caso del mapa de subte de la figura 1, la propiedad de ser una estación no puede ser atribuida sin atribuir simultáneamente relaciones espaciales, como conectividad. Así, por ejemplo, el mapa representa INDEPENDENCIA como una ESTACIÓN, pero también como CONECTADA a MORENO y SAN JUAN. Por ello, los predicados cartográficos son analógicos, en el sentido de Dretske. ${ }^{12}$

Sin embargo, vimos también que los mapas poseen elementos caracterizados por funciones semánticas distintivas, i.e. elementos generales (o predicados) y elementos singulares (o nombres), los cuales son capaces de representar información de una manera arbitraria, abstracta y selectiva. Los distintos ejemplares de $\mathrm{O}$, por caso, tienen la función de atribuir la propiedad de ser una estación, cumpliendo una función predicativa. Si bien es cierto que cuando un ejemplar de ese tipo es colocado en un mapa para representar una estación no puede hacerlo sin transmitir al mismo tiempo información acerca de las relaciones espaciales que la estación tiene con otras estaciones y líneas, aun así representa de una manera precisa la propiedad de ser una estación; esto es, sin hacer referencia a otras propiedades físicas, visuales, e incluso métricas, que una estación pudiera tener (por ejemplo, de qué material está hecha, que es subterránea, la forma, el color y la dimensión del edificio, etc.). En este sentido, estos símbolos, así como otros elementos constitutivos del mapa, son semánticamente arbitrarios, pues su aporte semántico no depende de semejanza alguna con aquello que representan. Por otra parte, son abstractos, pues representan de manera general e idealizada una propiedad con independencia de instanciaciones particulares de esa propiedad. Y son selectivos, pues representan una propiedad de una manera precisa, sin tener en cuenta otras características particulares y relaciones de sus instanciaciones, excepto las relaciones espaciales en las que está inmersa. En este sentido, los elementos constitutivos de un mapa son digitales.

En un isomorfismo espacial, las reglas combinatorias de los sistemas cartográficos no son arbitrarias. Sin embargo, son precisas y abstractas. Por ello, es posible deter- 
minar con exactitud qué relaciones (i.e. predicados con aridad $>1$ ) son constitutivas del sistema. Así, por ejemplo, un mapa de subte representa relaciones topológicas pero no métricas, un mapa de ruta representa relaciones métricas, pero no físicas. Por esa razón, la información sobre las relaciones espaciales entre las entidades representadas también puede ser abstraída y digitalizada y, en consecuencia, puede ser formulada con precisión en términos lingüísticos.

Justamente, dado que los predicados cartográficos transmiten información digital, el contenido de un mapa puede ser traducido lingüísticamente. Sin embargo, los predicados cartográficos también transmiten información analógica en virtud de no poder representar una propiedad con independencia de las relaciones espaciales atribuidas por el sistema al que el mapa pertenece. En este sentido, la expresión lingüística de los predicados de un mapa no transmite, como el mapa, información sobre las relaciones espaciales de las entidades representadas. Así por ejemplo, podemos expresar lingüísticamente el contenido del mapa de metro diciendo: Independencia es una estación, Moreno es una estación, San Juan es una estación, y así sucesivamente. Pero al hacerlo, son dejadas de lado las relaciones topológicas entre estas estaciones, así como la disposición de otras estaciones, las líneas de subte y sus combinaciones, etc. No obstante, dado que las relaciones constitutivas del sistema pueden ser digitalizadas y, por tanto, expresadas lingüísticamente, esa información puede ser recuperada y expresada en otras oraciones. Particularmente, podemos especificar además que Independencia, Moreno y San Juan están conectadas, que Moreno y San Juan están conectadas por Independencia, que Independencia está en la línea C, etc. No obstante, al ganar precisión, las representaciones lingüísticas pierden la capacidad de síntesis característica de los mapas.

Estos procesos de traducción deberían resultarnos familiares. ${ }^{13}$ Podemos imaginar una situación en la que llegamos a la ciudad de Buenos Aires. Estamos en la Terminal y queremos ir a la típica calle Callao. A partir del mapa de subte, podemos planificar nuestro itinerario. Decidimos tomar la línea C a Constitución, hacer una combinación por Diagonal Norte, para tomar la línea D a Congreso. En efecto, podemos pensar o decir en voz alta estas indicaciones (también podemos representarlas en el lenguaje del pensamiento) y luego intentar memorizarlas e incluso se las podríamos transmitir a alguien, si nos preguntara. Algo similar nos ofrece la aplicación de Google Maps, que combina información en formato analógico (como las líneas de subte) y digital (como las instrucciones verbales), con el agregado de los horarios, la demora y las distancias que separan a una estación de otra, el costo de los pasajes, etc., dando como resultado una representación extremadamente híbrida (ver figura 5). Ahora bien, cualquiera sea el caso, en el proceso de traducción, sólo extraemos del mapa la información que nos resulta relevante para nuestros propósitos prácticos: las líneas de subte, la dirección y las combinaciones que nos llevaran a destino desde nuestro punto de partida. Pero al hacerlo, omitimos otra información, disponible en 
el mapa, que en otras ocasiones podría resultarnos también relevante; por ejemplo, saber que Lavalle está justo antes puede ser de utilidad para predisponernos a bajar en la de Diagonal Norte, saber que hay una combinación con la línea B, podría ser útil si quisiéramos ir desde Retiro hasta Medrano, etc. En este sentido, puede que la información extraída tras el proceso de traducción resulte demasiado pobre para representar el contenido completo del mapa: pensemos, por caso, si quisiéramos dibujar el mapa de subte - el total de las conexiones entre líneas y estaciones - a partir de las instrucciones de Google Maps o de las indicaciones verbales. Mi predicción es que necesitaremos una larga lista de especificaciones adicionales. Pero, aunque esta lista sea demasiado larga, no creo que sea imposible escribirla. Cabe destacar que, en la medida en que el vehículo se vuelve más digital y, por tanto, más preciso y más arbitrario, como es el caso los mapas de Google y más aun de las oraciones, es posible introducir otro tipo de información (horarios, costos, demoras, distancias), además de la información espacial específica de los típicos mapas de subte. De modo que un aumento en la precisión puede significar, como en estos casos, mayor capacidad expresiva.

$\mathrm{Si}$, como lo sostienen diversas teorías empíricas, nuestra cognición opera con mapas cognitivos, entonces es necesario caracterizar el modo en que las representaciones cartográficas se relacionan con las representaciones con formato lingüístico. En esta dirección, mi hipótesis es que es posible que los procesos computacionales de formación de creencias así como nuestras habilidades espaciales (que pueden incluir desde el conocimiento de nuestra ubicación en el espacio, de la disposición espacial de nuestra ciudad - esto es, la posición de los edificios emblemáticos, el nombre y la dirección de las calles, el recorrido y las paradas del transporte público hacia nuestro lugar de trabajo, las áreas peatonales, etc. - así como los procesos de toma de decisiones para la planificación de itinerarios, etc.) incluyan un proceso de traducción con características muy similares a las mencionadas arriba.

\section{Conclusión}

En este trabajo he defendido una concepción pluralista de los vehículos conceptuales. A diferencia de otras variantes, el pluralismo defendido aquí sostiene que nuestra cognición opera con representaciones con distintos formatos que no sólo coexisten sino que interactúan de modos muy interesantes.

Para ello, he tomado como punto de partida la tesis empírica de que nuestra cognición opera con representaciones mentales con distintos tipos de formato. Luego, he sostenido que los logros cognitivos centrales de los conceptos (Fodor 2007), tales como la composicionalidad, la racionalidad y la aspectualidad, pueden ser alcanzados por medio de representaciones cartográficas. En esta dirección, he seguido los 


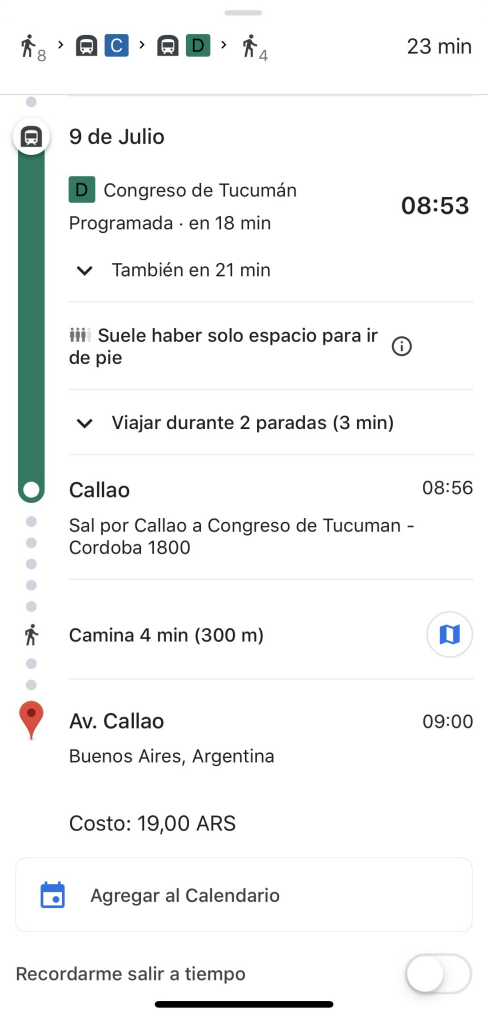

Figura 5: Extraído de la aplicación Google Maps

lineamientos de los trabajos de Camp y Rescorla, aunque con algunas divergencias importantes. A partir del análisis de ciertos rasgos formales de los mapas de subte, una variedad de sistema topológico, he sostenido que las representaciones con un formato cartográfico tienen una estructura composicional predicativa. Si este análisis se pudiera generalizar a los mapas cognitivos, como creo, se podría sostener que las representaciones con formato cartográfico pueden jugar el rol de los conceptos.

Finalmente, he analizado un modo en que pueden interactuar las representaciones cartográficas con las lingüísticas, en particular, cómo el contenido de los mapas puede ser traducido o expresado lingüísticamente. De acuerdo con mi análisis, gracias a un equilibrio entre la información digital y analógica, los mapas pueden representar mucha información espacial de un modo muy preciso, con un gran poder de síntesis y un alto poder de abstracción. Las representaciones con formato lingüístico, en cambio, logran expresar contenidos con mayor precisión, pero a costa de perder capacidad de síntesis. No obstante, a diferencia de los mapas, las oraciones pueden representar simultáneamente información de diversa índole (tales como relaciones 
topológicas y métricas, magnitudes numéricas, etc.) ganando capacidad expresiva.

Si bien está basado en una representación externa - un mapa de subte -, el análisis puede extenderse para integrar la hipótesis empírica de que nuestra cognición opera con representaciones con distintos tipos de formatos en el marco de una teoría de los conceptos. Este análisis, además, sugiere que el uso combinado de ambos tipos de representaciones podría enriquecer de diversas maneras nuestra vida cognitiva.

\section{Referencias bibliográficas}

Aguilera, M. 2016. Cartographic Systems and Non-linguistic Inference. Philosophical Psychology 29(3): 349-364.

Aguilera, M.; Castellano, F. (manuscrito). Maps, Language, and the Conceptual-Nonconceptual Distinction.

Aguilera, M.; Castellano, F. (manuscrito). Heterogeneous inference with maps. 2018 - Meeting of the Society of Philosophy and Psychology. Michigan.

Beck, J. 2013. Why We Can't Say What Animals Think. Philosophical Psychology 26: 520-546. Bermúdez, J. L. 1998. The Paradox of Self Consciousness. Cambridge: The MIT Press.

Blumson, B. 2012. Mental Maps. Philosophy and Phenomenological Research 85(2): 413-434.

Braddon-Mitchell, D.; Jackson, F. 1996. Philosophy of Mind and Cognitive Science. Cambridge: Blackwell Publishers.

Bronner, B. 2015. Maps and Absent Symbols. Australasian Journal of Philosophy 93(1): 4359.

Burge, T. 2010. Origins of Objectivity. Oxford: Oxford University Press.

Camp, E. 2004. The Generality Constraint and Categorial Restrictions. The Philosophical Quarterly 54(215): 209-231.

Camp, E. 2007. Thinking With Maps. Philosophical Perspectives 21: 145-182.

Camp, E. 2009. A Language of Baboon Thought? En R. Lurz (Ed.), The Philosophy of Animal Minds, pp.108-117. Cambridge: Cambridge University Press.

Camp, E. 2015. Logical Concepts and Associative Characterizations. En E. Margolis \& S. Laurence (Edits.), The Conceptual Mind: New Directions in the Study of Concepts,pp. 591-621. MIT Press.

Camp, E. 2018. Why Maps are Not Propositional. En A. Grzankowski \& M. Montague (Edits.), Non-Propositional Intentionality. Oxford University Press.

Carey, S. E. 1985. Conceptual change in childhood. Cambridge: The MIT Press.

Carey, S. 2009. The origin of concepts. Oxford: Oxford University Press.

Casati, R.; Varzi, A. C. 1999. Parts and Places. The Structures of Spatial Representation. Cambridge: The MIT Press.

Clapp, L. 2012. Is even thought compositional? Philosophical Studies 157(2): 299-322.

Dretske, F. 1981. Sensation and Perception. In Y. H. Gunther, Essays on Nonconceptual Content, pp.25-41. Cambridge: The MIT Press.

Dretske, F. 2000. Simple Seeing. En F. Dretske, Perception, Knowledge and Belief, pp.97-112. Cambridge: Cambridge University Press. 
Epstein, R. A.; Patai, E. Z.; Julian, J. B.; Spiers, H. J. 2017. The cognitive map in humans: spatial navigation and beyond. Nature Neuroscience 20(11): 1504-1513.

Fodor, J. 1975. The Language of Thought. Cambridge, Massachusetts: Harvard University Press.

Fodor, J. 1995. Concepts; A potboiler. Philosophical Issues 6: 1-24.

Fodor, J. 1998. Conceptos. Donde la ciencia cognitiva se equivocó. Barcelona: Gedisa.

Fodor, J. 2001. Language, Thought and Compositionality. Mind and Language 16: 1-15.

Fodor, J. 2007. The revenge of the given. En B. McLaughlin \& J. Cohen, Contemporary debates in philosophy of mind, pp.105-116. Singapore: Blackwell.

Fodor, J. 2008. The Language of Thought Revisited. Oxford: Oxford University Press.

Fodor, J.; Pylyshyn, Z. 1988. Connectionism and Cognitive Architecture. Cognition 28: 3-71.

Haimovici, S. 2018. The Modal-Amodal Distinction in the Debate on Conceptual Format. Philosophies 3(2): 7.

Heck, R. K. 2007. Are There Different Kinds of Content? En B. P. McLaughlin \& J. Cohen (Edits.), Contemporary Debates in Philosophy of Mind, pp.117-138. Oxford: Blackwell.

Hills, T. T.; Jones, M. N.; Todd, P. M. 2012. Optimal foraging in semantic memory. Psychological review 119: 431-440.

Johnson, K. 2004. On the Systematicity of Language and Thought. The Journal of Philosophy 101: 111-139.

Johnson, K. 2015. Maps, languages, and manguages: Rival cognitive architectures? Philosophical Psychology 28(6): 815-836.

Kamil, A. C.; Jones, J. E. 1997. The seed-storing corvid Clark's nutcracker learns geometric relationships among landmarks. Nature 390: 276-279.

Kulvicki, J. 2015. Maps, Pictures, and Predication. Ergo 2(7): 149-174.

Laurence, S.; Margolis, E. 1999. Concepts and Cognitive Sciences. En E. Margolis \& S. Laurence (Edits.), Concepts: Core Readings, pp.3-81. Cambridge: The MIT Press.

Machery, E. 2009. Doing without Concepts. Oxford: Oxford University Press.

Margolis, E.; Laurence, S. 2003. Concepts. En S. Stich \& T. Warfield (Edits.), The Blackwell Guide to the Philosophy of Mind, pp.190-213. Oxford: Blackwell.

McCaffrey, J. 2015. Reconceiving conceptual vehicles: Lessons from semantic dementia. Philosophical Psychology 28(3): 337-354.

O'Keefe, J.; Nadel, L. 1978. The hippocampus as a cognitive map. Oxford: Clarendon Press.

Peacocke, C. 1992a. A Study of Concepts. Cambridge: The MIT Press.

Peacocke, C. 1992b. Scenarios, concepts, and perception. En Y. H. Gunther (Ed.), Essays on Nonconceptual Content, pp.107-132. MIT Press.

Prinz, J. 2002. Furnishing the Mind. Concepts and their perceptual basis. Cambridge: The MIT Press.

Recabarren, N. (manuscrito). ¿Multiplicidad de contenidos? $2^{\circ}$ Coloquio sobre conceptos y percepción. Villa General Belgrano.

Recanati, F. 2012. Compositionality, Semantic Flexibility, and Context-Dependence. En W. Hinzen, E. Machery, \& M. Werning (Edits.), The Oxford Handbook of Compositionality, pp.175-191. Oxford: Oxford University Press.

Rescorla, M. 2009a. Chrysippus' dog as a case study in non-linguistic cognition. En R. Lurz (Ed.), The Philosophy of Animal Minds, pp.52-71. Cambridge: Cambridge University Press. 
Rescorla, M. 2009b. Cognitive Maps and the Language of Thought. The British Journal for the Philosophy of Science 60: 377-407.

Rescorla, M. 2009c. Predication and cartographic representation. Synthese 169: 175-179.

Rescorla, M. 2018. Maps in the head? En K. Andrews \& J. Beck (Edits.), The Routledge handbook of philosophy of animal minds, pp.34-45. New York: Routledge.

Shea, N. 2014. Exploitable Isomorphism And Structural Representation. Proceedings of the Aristotelian Society XCIV: 123-144.

Taylor, K. A. 2010. On Singularity. En New Essays on Singular Thought, pp. 77-102. Oxford: Oxford University Press.

Tolman, E. 1948. Cognitive maps in rats and men. The Psychological Review 55: 189-208.

Travis, C. 2008. On Constraints of Generality. En Ocassion - Sensitivity, pp.271-289. Oxford: Oxford University Press.

Tversky, B.; Lee, P. U. 2014. Pictorial and Verbal Tools for Conveying Routes. Lecture Notes in Computer Science, pp.51-64, vol 1661. Berlin, Heidelberg: Springer.

Weiskopf, D. 2009a. The plurality of concepts. Mind 169: 145-173.

Wittgenstein, L. [1921] 2003. Tractatus logico-philosophicus. Madrid: Alianza Editorial.

\section{Notes}

${ }^{1}$ Se podría señalar que la noción de mapa usada en la psicología y neurociencia se refiere al contenido de las representaciones mentales, pero no a su formato. Se trata de una cuestión importantísima que, sin embargo, no puedo resolver aquí. Seguiré en cambio, a Rescorla (2018) y asumiré que las investigaciones empíricas sobre los mapas cognitivos dan lugar a una caracterización de mapa cognitivo en términos de las propiedades formales o estructurales de los mapas.

${ }^{2}$ Johnson (2015) sostiene que hasta ahora no se ha mostrado que los mapas difieran significativamente del lenguaje. Particularmente, dice que no hay diferencias computacionales entre pensar con mapas y pensar con palabras. Entre sus argumentos principales, sostiene que las restricciones semánticas de los mapas, que suelen evocarse para diferenciarlos del lenguaje, son estipulativas. Sin embargo, mientras que es cierto que es arbitrario el tipo de isomorfismo que ha de explotar un sistema representacional, una vez que esto es establecido, tales restricciones resultan constitutivas. En este sentido, si un sistema explota relaciones topológicas, por definición no podrá explotar relaciones métricas y así sucesivamente.

${ }^{3} \mathrm{Si}$ bien este trabajo está centrado en el papel de representaciones cartográficas, no descarto que otros tipos de representaciones puedan jugar el rol de los conceptos. Sin embargo, dado que el espectro de representaciones no-lingüísticas es demasiado amplio y heterogéneo, el análisis de otros tipos de representaciones excede este trabajo. Mi idea precisamente es rechazar la dicotomía entre representaciones lingüísticas y no lingüísticas, que Fodor (2008) propuso para diferenciar las representaciones conceptuales de las no conceptuales. Para ello, es preciso estudiar separadamente las particularidades de cada tipo de sistema. En principio, tomando los criterios de Fodor (2007), si una representación tiene una estructura predicativa y composicional adecuada para los procesos inferenciales, entonces puede ser un concepto. Esto deja fuera las imágenes (figura 2) así como otras representaciones de magnitud analógica (Beck 2013). Ver también mi XXXX. 
${ }^{4}$ De este modo, la composicionalidad se encuentra ligada al atomismo semántico y a la teoría informacional, según la cual, el contenido de un concepto está determinado por la clase de entidades a las que se refiere.

${ }^{5}$ Una consecuencia que se sigue de esta forma de explicar la aspectualidad es que dos conceptos co-referenciales tienen el mismo contenido. Ello impide explicar, entre otras cosas, los casos de Frege, esto es, casos en los que la identidad de los referentes (i.e. Mary Shelley y quien escribió Franquestein) es desconocida para un sujeto. Para subsanar este inconveniente, Fodor sostiene que los conceptos se individúan por su referente y forma sintáctica, alejándose de esta manera de la tradición fregeana que traza esa distinción en términos de los sentidos o significado cognitivo (Prinz 2002; Schneider 2011).

${ }^{6}$ Para la idea de explotar un isomorfismo, ver Shea (2014).

${ }^{7}$ En (Aguilera 2016) he sugerido cómo se podría diluir esta intuición, representando de manera explícita la negación y, por ende, la ausencia, pero sin adoptar un compromiso con la noción de predicado provista por la lógica de primer orden.

${ }^{8}$ Johnson (2004) y Clapp (2012) cuestionan la viabilidad de una concepción mixta de este tipo, pues niegan que se pueda mantener que el pensamiento sea sistemático si el lenguaje natural no lo es.

${ }^{9}$ Hasta qué punto son irrestrictas las posibilidades de combinación de los conceptos es un tema de abundante discusión. Fodor, al igual que Evans (1982) y otros, consideran que hay restricciones categoriales, metafísicamente fundamentadas (ver Fodor (2007). Sin embargo, desde un punto de vista sintáctico, las restricciones sólo competen al rol de los conceptos involucrados, si es un término singular o general, por ejemplo. Éste es el tipo de restricción que me interesa resaltar aquí. Mientras que las restricciones sintácticas en los sistemas lingüísticos son mínimas en este sentido, los mapas en cambio, tienen importantes limitaciones. Por empezar, no pueden representar relaciones no espaciales ni propiedades a menos que éstas sean atribuidas a entidades particulares. Por cuestiones de espacio, no puedo argumentar esto aquí.

${ }^{10}$ La distinción entre analógico y digital ha sido entendida de diferentes maneras. Por ejemplo, Goodman (1976) sostiene que una representación es analógica si es densa, esto es, si hay una tercera representación entre dos representaciones cualesquiera. Si es discreta, entonces la representación es digital. Sin embargo, esta distinción no permite capturar la idea de que las representaciones cartográficas no pueden representar entidades sin atribuirle relaciones espaciales, lo cual es independiente de su densidad. La distinción ofrecida por Beck (2012), en cambio, se centra en el carácter isomorfo de las representaciones analógicas. En este sentido, puede que sea compatible con la posición defendida aquí, aunque no explota algunas de las consecuencias que considero que se siguen de ello.

${ }^{11}$ Por supuesto que las emisiones lingüísticas transmiten también información sobre su emisor - tales como su identidad, su estado de ánimo e intenciones - y sobre el canal de emisión. Sin embargo, el foco de este trabajo está puesto en el formato de la representación lingüística, abstraído de los elementos pragmáticos que pudieran tener lugar en las preferencias particulares.

${ }^{12}$ Es importante señalar que hay otros modos de emplear la noción de analogicidad. En particular, puede emplearse como parte de un argumento a favor del no conceptualismo, basado en la especificidad del contenido (Dretske 2000; Heck 2007). Aquí, no obstante, la estoy utilizando de una manera diferente, aludiendo a la propiedad de ciertos predicados, P, para 
portar información conceptual más allá de la representada estrictamente por el predicado mismo, $\mathrm{P}$.

${ }^{13}$ Tversky y Lee (2014) sostienen que las instrucciones lingüísticas, como las direcciones, y las instrucciones pictóricas, como los mapas y dibujos, sirven como herramientas para trazar rutas, compuestas de elementos comunes, lo cual explicaría la naturalidad con la que pasamos de un sistema a otro.

\section{Agradecimientos}

He presentado partes de este trabajo en el Seminario Seminario María Zambrano, Universidad de Barcelona, Barcelona, 2019. Quisiera agradecer a la audiencia por sus comentarios y, en especial, a Manuel Pérez Otero. Por otro lado, quisiera reconocer a Mariela Destéfano; su lectura y sus sugerencias fueron centrales para esta versión. También, agradezco a Daniel Kalpokas, por sus comentarios a versiones tempranas del trabajo, así como también a los referís anónimos, por sus sugerencias en el tramo final. Para este trabajo, he contado con el financiamiento de FONCYT. 